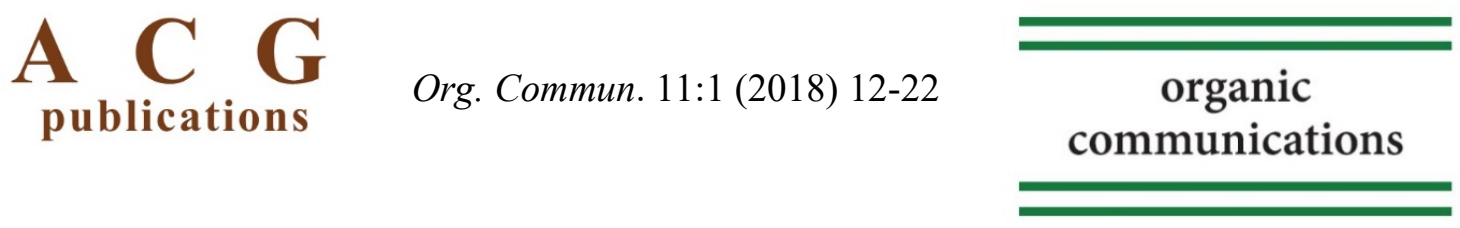

\title{
Triarylborane functionalized selenophenothiophene analogues: Syntheses, photophysical and electrochemical properties
}

\author{
Gulsen Turkoglu* \\ Department of Chemistry, Istanbul Technical University, Maslak, Istanbul, Türkiye
}

(Received December 12, 2017; Revised January 12, 2018; Accepted January 18, 2018)

\begin{abstract}
Two novel acceptor-donor-acceptor (A-D-A) small molecules BSeT1 and BSeT2 containing dimesitylborane as an electron acceptor and selenopheno[3,2-b]thiophene and selenopheno[2,3-b]thiophene as electron donors linked through a thiophene $\pi$-conjugated spacer were synthesized and characterized. Their photophysical and electrochemical properties were investigated. In particular, BSeT1 demonstrated that the presence of a conjugated selenopheno[3,2-b]thiophene causes a bathochromic shift of the absorption bands with respect to a cross conjugated selenopheno[2,3- $b$ ]thiophene in BSeT2. The fluorescence spectra of BSeT1 exhibited yellow emission at 516 and $543 \mathrm{~nm}$, while BSeT2 showed blue emission peak of $468 \mathrm{~nm}$. Also, the emission quantum efficiency of BSeT1 was determined to be up to $11 \%$ in THF. These compounds demonstrated red-shift emissions in their solid states compared to those in organic solvents. The optimized geometries of BSeT1 and BSeT2 were achieved by density functional theory (DFT) to examine their electronic structures, frontier molecular orbital (FMO) energy levels and electronic excitations. The comparisons illustrated that the theoretical data are well in alignment with the corresponding experimental results. In addition, the structure-property relationships of BSeT1 and BSeT2 were assessed.
\end{abstract}

Keywords: Selenophenothiophene; triarylborane; UV-Vis absorption spectroscopy; fluorescence; density functional theory (DFT). C2018 ACG Publication. All right reserved.

\section{Introduction}

Like fused thienothiophenes (TTs) and dithienothiophenes (DTTs), ${ }^{1}$ fused selenophene ring compounds have attracted much interest due to their promising use in optoelectronic materials. ${ }^{2,3}$ They are useful synthetic intermediates as building blocks to produce selenophene ring containing oligomers and polymers. ${ }^{4}$ In addition, conjugated organic materials with Se atom are expected to have higher lying highest occupied molecular orbital (HOMO) and lowest unoccupied molecular orbital (LUMO) energy levels resulting in lower oxidation and reduction potentials, and easier polarizability. Owing to the presence of intermolecular Se $\cdots$ Se interactions, they lead to plausible inter-chain charge transfers, ${ }^{5}$ accepted as promising properties for optoelectronics. Additionally, fused ring systems are believed to supply a high degree of planarity and rigidity rendering higher crystallinity, extended pi overlap and improved mobility. As a result, an overall improvement of device performance is achieved. Hetero aromatic fused selenophene derivatives afford high charge mobilities emerging from strong intermolecular interactions via Se atoms. ${ }^{6,7}$ While thiophene containing systems have thoroughly been investigated, their selenium analogues have lately attracted the interest of scientists for their potential applications in electronic field. Replacement of sulfur with selenium, recognized as a lower

\footnotetext{
* Corresponding author: E-Mail: gulsent06@hotmail.com The article was published by ACG Publications www.acgpubs.org/OC/ index.htm (C) January-March 2018 EISSN:1307-6175 DOI: http://doi.org/10.25135/acg.oc.34.17.12.061
} 
electronegative and more polarizable atom with respect to sulfur, influences the electronic and optical properties. ${ }^{8-10}$

The conjugated organic small molecules in material science can be successfully achieved through $\mathrm{D}-\pi-\mathrm{A}$ approach, which requires electron donor and strong acceptor units. $^{11,12}$ In D-A type molecules, LUMO is predominantly located on the electron deficient unit, whereas HOMO is mainly found on the electron donor moiety. In addition, conjugated organic molecules and polymers having trivalent boron have also attracted great attention owing to their excellent optoelectronic properties. ${ }^{13-16}$ Triarylborane has a trigonal planar $\mathrm{sp}^{2}$ hybridized three-coordinate boron atom with a vacant $\mathrm{p}$ orbital in the electronic ground state. ${ }^{17}$ Hence, triarylboranes have an inherently electron deficient property, and they are, thus, used as an electron acceptor as well as Lewis acid in D-A type organic $\pi$-conjugated systems ${ }^{16}$. Empty p-orbital of boron can facilitate influential electronic delocalization with linked $\pi$-conjugated units. Also, the steric hindrance around the boron centre generated by the bulky groups like mesityl was provided to be a useful way to prepare air-stable triarylboranes. ${ }^{16}$ These compounds with extended $\pi$-conjugate systems afford $\mathrm{p}_{\pi}-\pi^{*}$ conjugation effectively, which is reflected as characteristic absorption and high emission properties. ${ }^{18}$ However, triarylboranes are highly luminescent materials in solid state, and they have good electrochemically reversible reduction potentials. In addition, while LUMO energy levels of boron based conjugated compounds are relatively low due to good electron affinity, ${ }^{16,19,20}$ the HOMO levels of boron materials are tuneable with electron donor groups. Consequently, diminishing LUMO energy level influences HOMO-LUMO gap and thus photophysical properties of the compounds, making the boron based $\pi$-conjugated systems better electron acceptors. ${ }^{21}$ As a result, their high HOMO and low LUMO energy levels can improve the carrier-injecting properties and thereby the device performance. Also, triarylborane based D-A type molecules with great electronic dipoles support hole-electron charge transfers depending on excitation. ${ }^{22,23}$

We previously reported the synthesis of selenophenothiophene (SeT) analogues containing mesitylboron copolymers. ${ }^{24}$ Herein, we present the synthesis, optical and electrochemical properties of two novel A- $\pi-\mathrm{D}-\pi-\mathrm{A}$ type BSeT1 and BSeT2 compounds, possessing dimesityl boron acceptor units, and conjugated and cross conjugated fused SeT donor systems linked through a thiophene $\pi$ spacer. Their UV-Vis, florescence and electrochemical properties were investigated. Furthermore, structure-property relationship of them was addressed in depth.

\section{Experimental}

\subsection{Chemical Material and Apparatus}

All reagents were used as received from commercial sources without further purification. Tetrahydrofuran (THF) and diethyl ether were dried over sodium in presence of benzophenone. All the reactions were performed under $\mathrm{N}_{2}$. 2,5-Di(thiophen-2-yl)selenopheno[3,2- $\left.b\right]$ thiophene (1) and 2,5di(thiophen-2-yl)selenopheno[2,3-b]thiophene (2) were synthesized according to our previous work. ${ }^{24}$

${ }^{1} \mathrm{H}$ and ${ }^{13} \mathrm{C}$ NMR spectra were recorded on a Varian model Agilent VNMRS $500 \mathrm{MHz}$ NMR spectrometer and the chemical shifts are reported in ppm downfield from tetramethylsilane (TMS). UV-Vis spectra were acquired on a HITACHI U-0080D spectrophotometer. Fluorescence spectra were recorded on a HITACHI F-4500 fluorescence spectrophotometer. The fluorescence quantum efficiency of the compounds were determined in dilute THF by using Coumarin 1 and Coumarin 6 $\left(\Phi_{F}=0.73\right.$ and 0.78 in ethanol, respectively) as standard substances applying the previously described methods. ${ }^{39,40}$ Cyclic voltammetry (CV) experiments were carried out as potentiostat a CH-Instruments Model 400A instrument. Oxidation and reduction processes of the compounds were measured in dry $\mathrm{CH}_{2} \mathrm{Cl}_{2}$ solution $\left(1 \times 10^{-3} \mathrm{M}\right)$ with $0.1 \mathrm{M}$ tetrabutylammonium hexafluorophosphate $\left(n-\mathrm{Bu}_{4} \mathrm{NPF}_{6}\right)$ as the supporting electrolyte at room temperature under $\mathrm{N}_{2}$. A scan rate of $100 \mathrm{mV} \mathrm{s}^{-1}$ was adopted in all the measurements. A standard three-electrode system was used for the measurements: platinum wires as a working and counter electrodes and $\mathrm{Ag}$ wire as the reference electrode. A ferrocene internal standard was used to calibrate the results. 


\subsection{Synthesis of (5,5'-(selenopheno[3,2-b] thiophene-2,5-diyl)bis(thiophene-5,2-diyl))- bis(dimesitylborane) (BSeT1)}

To a solution of compound $1(235 \mathrm{mg}, 700 \mu \mathrm{mol})$ in dry THF $(30 \mathrm{~mL})$ was added $600 \mu \mathrm{L}$ of $n$-BuLi (2.5 M, $1.47 \mathrm{mmol})$ dropwise at $-78^{\circ} \mathrm{C}$ under nitrogen atmosphere. The mixture was stirred at $-78{ }^{\circ} \mathrm{C}$ for 2 hours and $394 \mathrm{mg}(1.47 \mathrm{mmol})$ of dimesitylboron fluoride was added quickly to the solution. The mixture was allowed to warm up to room temperature and it was stirred for 2 days. Then, it was poured into $100 \mathrm{~mL}$ of distilled water and extracted with DCM $(3 \times 20 \mathrm{~mL})$. After removal of solvent, the product was purified through a column chromatography over silica gel using $n$ hexane/DCM (5:1) as eluent furnishing BSeT1 as an orange-red solid in 21\% (120 mg) yield. $\mathrm{R}_{f}: 0.62$ ( $n$-hexane/DCM, 5:1). mp: 156-158 ${ }^{\circ} \mathrm{C} .{ }^{1} \mathrm{H}$ NMR $\left(500 \mathrm{MHz}, \mathrm{CDCl}_{3}\right) \delta 7.45(\mathrm{~s}, 1 \mathrm{H}), 7.42(\mathrm{~s}, 1 \mathrm{H}), 7.36$ $(\mathrm{d}, J=4.0 \mathrm{~Hz}, 1 \mathrm{H}), 7.32(\mathrm{~d}, J=4.0 \mathrm{~Hz}, 1 \mathrm{H}), 7.15(\mathrm{~d}, J=3.5 \mathrm{~Hz}, 1 \mathrm{H}), 7.02(\mathrm{dd}, J=5.0,3.5 \mathrm{~Hz}, 1 \mathrm{H})$, $6.84(\mathrm{~s}, 8 \mathrm{H}), 2.32(\mathrm{~s}, 12 \mathrm{H}), 2.16(\mathrm{~s}, 24 \mathrm{H}) .{ }^{13} \mathrm{C}$ NMR $\left(126 \mathrm{MHz}, \mathrm{CDCl}_{3}\right): \delta 156.0,155.9,147.8,143.9$, $140.7,140.4$, 131.5, 129.7, 129.5, 129.3, 128.2 , 127.3, 126.8, 126.7, 120.3, 119.7, 119.2, 114.6, 23.5, 21.3

\subsection{Synthesis of (5,5'-(selenopheno[2,3-b] thiophene-2,5-diyl)bis(thiophene-5,2-diyl))- bis(dimesitylborane) (BSeT2)}

Compound BSeT2 was obtained in a similar way as BSeT1. In this reaction, compound 2 (336 $\mathrm{mg}, 960 \mu \mathrm{mol}), n$-BuLi $(830 \mu \mathrm{L}, 2.5 \mathrm{M}, 2.10 \mathrm{mmol})$, dimesitylboron fluoride (562 mg, $2.10 \mathrm{mmol}$ ) were reacted in $50 \mathrm{~mL}$ of dry THF. BSeT2 was obtained as an orange solid with a yield of $15 \%$ (132 mg). $\mathrm{R}_{f}: 0.54$ ( $n$-hexane/DCM, 5:1). mp: $172{ }^{\circ} \mathrm{C} .{ }^{1} \mathrm{H}$ NMR $\left(500 \mathrm{MHz}, \mathrm{CDCl}_{3}\right) \delta 7.48(\mathrm{~s}, 1 \mathrm{H}), 7.40(\mathrm{~d}$, $J=1.0 \mathrm{~Hz}, 1 \mathrm{H}), 7.36(\mathrm{~d}, J=2.5 \mathrm{~Hz}, 1 \mathrm{H}), 7.34(\mathrm{~d}, J=2.5 \mathrm{~Hz}, 1 \mathrm{H}), 7.22(\mathrm{~d}, J=4.0 \mathrm{~Hz}, 1 \mathrm{H}), 7.02(\mathrm{dd}$, $J=5.0,3.5 \mathrm{~Hz}, 1 \mathrm{H}), 6.82(\mathrm{~s}, 8 \mathrm{H}), 2.31(\mathrm{~s}, 12 \mathrm{H}), 2.15(\mathrm{~s}, 24 \mathrm{H}) .{ }^{13} \mathrm{C}$ NMR $\left(126 \mathrm{MHz}, \mathrm{CDCl}_{3}\right): \delta 152.3$, $149.1,148.1,147.1,146.6,140.7,139.7,138.6,134.2,132.6,129.2,128.1,127.4,126.0,125.2,122.6$, 23.1, 21.2.

\section{Results and Discussion}

\subsection{Synthesis and Characterization}

The syntheses of the A-D-A type compounds BSeT1 and BSeT2 are shown in Scheme 1. 2,5-Di(thiophen-2-yl)selenopheno[3,2-b]thiophene (1) and 2,5-di(thiophen-2-yl)selenopheno[2,3-b]thiophene (2) were synthesized according to our previous work. ${ }^{24}$ Lithiation of the compound $\mathbf{1}$ with $n$-BuLi at $-78{ }^{\circ} \mathrm{C}$ was followed by addition of $\mathrm{Mes}_{2} \mathrm{BF}$ in THF at room temperature providing the compound BSeT1 as a red solid in 20\% yield. The compound BSeT2 was obtained in $15 \%$ yield by successive treatment of 2 with $n$ - $\mathrm{BuLi}-78{ }^{\circ} \mathrm{C}$ and $\mathrm{Mes}_{2} \mathrm{BF}$ at room temperature in THF.

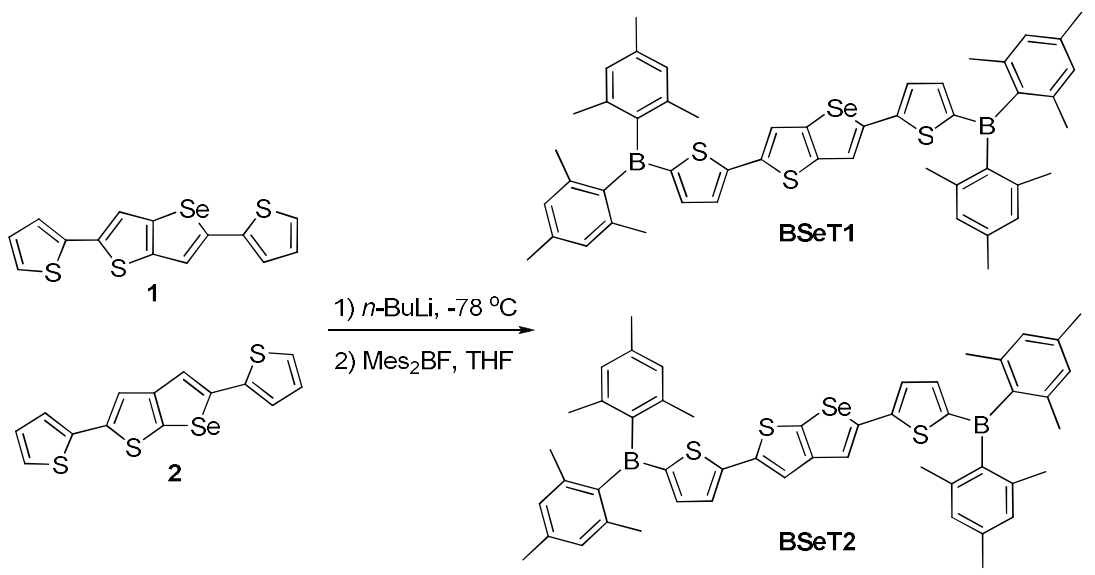

Scheme 1. Synthetic route for BSeT1 and BSeT2 compounds 
Compounds were characterized by NMR spectroscopy. In ${ }^{1} \mathrm{H}$ NMR spectra, the presence of four doublets for SeT units confirms the existing of the unsymmetrical structures. The chemical shifts of the aromatic protons were recorded to be between 7.45 and $6.84 \mathrm{ppm}$ as expected. While metaprotons of dimesitylboron units were observed as singlets at $6.84 \mathrm{ppm}$ for BSeT1 and $6.82 \mathrm{ppm}$ for BSeT2 as anticipated, ortho-methyl groups $\left(-\mathrm{CH}_{3}\right)$ were detected in the upfield at 2.32 and $2.31 \mathrm{ppm}$, and para-methyl groups at $2.16 \mathrm{ppm}$ and $2.15 \mathrm{ppm}$, respectively. While para- $\mathrm{CH}_{3}$ carbon atoms of dimesitylboron were observed in the range of 23.5 and $23.1 \mathrm{ppm}$ in ${ }^{13} \mathrm{C} \mathrm{NMR}$, ortho- $\mathrm{CH}_{3}$ groups appeared at $21.2 \mathrm{ppm} .{ }^{1} \mathrm{H}$ NMR and ${ }^{13} \mathrm{C}$ NMR spectra confirm the validity of the structures given in Scheme 1.

\subsection{Absorption Spectra}

The UV-Vis absorptions of BSeT1 and BSeT2 in THF are illustrated in Figure 1 and their photophysical data are summarized in Table 1 . The absorption peaks at 340 and $335 \mathrm{~nm}$ originate from $\pi-\pi^{*}$ transitions of SeT units, whereas the longer wavelength absorption maxima at 388 and $442 \mathrm{~nm}$ emerging from $\pi-\pi^{*}$ transitions of acceptor unit in the whole molecule. BSeT1 exhibited the longestwavelength absorption maximum at $442 \mathrm{~nm}$, indicating a red-shift of $54 \mathrm{~nm}$ risen from $\pi$-conjugated $\mathrm{SeT}$. This low-energy band was appointed to intramolecular charge transfer (ICT) from the conjugated $\mathrm{SeT}$ core to the two dimesitylboron terminals. However, BSeT2 possessing cross conjugated SeT was observed to have the longer wavelength absorption maximum of $388 \mathrm{~nm}$, corresponding to a low charge transfer band. The hypsochromic shift is consistent with a reduce in the conjugation length and increase in the HOMO-LUMO band gap of BSeT2 due to the cross-conjugated SeT unit hampering full delocalization over the whole planar framework. Moreover, the cross-conjugated system in BSeT2 lowers the HOMO energy level and thereby increases the ionization potential. In addition, BSeT1 and BSeT2 showed slightly blue shift by $2 \mathrm{~nm}$ and $24 \mathrm{~nm}$ in THF respectively, compared to their thienothiophene derivatives. ${ }^{25}$ Thus, compared to the sulfur structural analogues, ${ }^{25}$ this can be explained by the presence of "Se" atom in the ring. The optical band gaps, calculated from the onset of the absorption spectra, were recorded to be 2.43 and $2.60 \mathrm{eV}$ for BSeT1 and BSeT2, respectively.

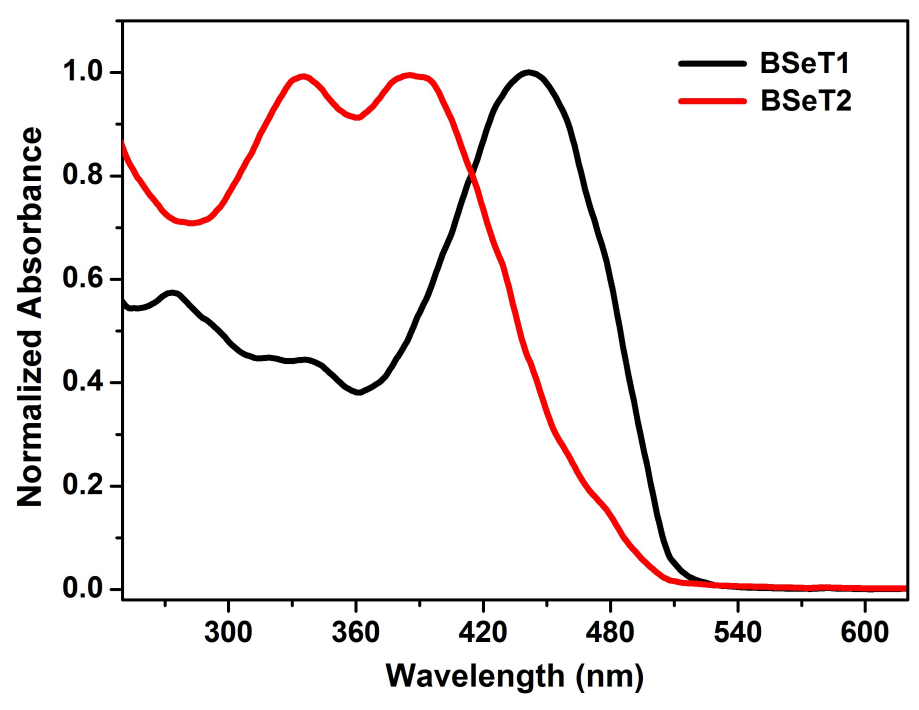

Figure 1. UV-Vis absorption spectra of BSeT1 and BSeT2 in THF 
Table 1. Photophysical and electrochemical properties of BSeT1 and BSeT2

\begin{tabular}{|c|c|c|c|c|c|c|c|}
\hline Comp. & $\begin{array}{l}\lambda_{\text {abs }}{ }^{a} \\
(\mathrm{~nm})\end{array}$ & $\begin{array}{l}\lambda_{\mathrm{em}}{ }^{\mathrm{a}} \\
(\mathrm{nm})\end{array}$ & $\Phi_{F}$ & $\begin{array}{l}\lambda_{\text {em }}{ }^{e} \\
(\mathrm{~nm})\end{array}$ & \multicolumn{2}{|c|}{$\begin{array}{c}\Delta \tilde{v}^{f} \\
\left(\mathbf{c m}^{-1}\right)\end{array}$} & $\mathbf{E}_{\mathrm{g}}^{\text {opt }}$ \\
\hline BSeT1 & 442,340 & $516,543(\mathrm{~s})^{b}$ & $0.11^{\mathrm{c}}$ & 546 & \multicolumn{2}{|r|}{3200} & 2.43 \\
\hline BSeT2 & 388,335 & $468,491(\mathrm{~s})$ & $0.08^{\mathrm{d}}$ & 480 & \multicolumn{2}{|r|}{4400} & 2.60 \\
\hline Comp. & \multicolumn{2}{|c|}{$\begin{array}{c}\mathbf{E}^{\mathbf{o x}}{ }_{\text {onset }} / \mathbf{E}^{\text {red }}{ }_{\text {onset }} \\
\text { (V) }\end{array}$} & $\begin{array}{c}\text { HOMO }^{g} \\
(\mathrm{eV})\end{array}$ & $\begin{array}{c}\text { LUMO }^{\text {g }} \\
(\mathrm{eV})\end{array}$ & $\begin{array}{l}\mathbf{E}_{\mathrm{g}}^{\mathrm{CV}} \\
(\mathrm{eV})\end{array}$ & \multicolumn{2}{|c|}{$\begin{array}{c}\text { FMO calc. }^{\mathrm{h}} \\
(\mathrm{eV})\end{array}$} \\
\hline BSeT1 & \multicolumn{2}{|c|}{$0.68 /-1.38$} & -5.08 & -3.02 & 2.06 & \multicolumn{2}{|c|}{$-5.24 /-2.48$} \\
\hline BSeT2 & \multicolumn{2}{|c|}{$0.89 /-1.56$} & -5.29 & -2.84 & 2.45 & \multicolumn{2}{|c|}{$-5.40 /-2.20$} \\
\hline 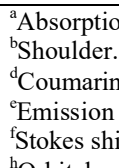 & $\begin{array}{l}\text { and emission } \\
\text { luorescence } \mathrm{q} \\
\text { as the standa } \\
\text { axima of the } n \\
{ }^{\mathrm{g}} \mathrm{HOMO}=-\end{array}$ & $\begin{array}{l}\text { upon excitation } \\
\text { yields calculated } \\
\text { hanol. } \\
\text { e films coated on } \\
\mathrm{E}^{\mathrm{o}} \mathrm{E}_{\text {onset }}(\mathrm{eV}) \mathrm{g}^{\mathrm{g}} \mathrm{LU}\end{array}$ & $\begin{array}{l}\text { e absorption maxi } \\
\text { ng Coumarin } 6 \text { an } \\
\text { s. } \\
=-\left(4.40+E^{\text {red }} \text { ons }\right.\end{array}$ & & & & \\
\hline
\end{tabular}

\subsection{Fluorescence Spectra}

Fluorescence spectra of BSeT1 and BSeT2 were recorded in THF and as solid state films (Figure 2 and Table 1). To determine the wavelength of emission, each molecule was excited at the wavelength of the lower energy absorption band. BSeT1 was revealed as green emission with an intense band at $516 \mathrm{~nm}$ and a lower energy shoulder at $543 \mathrm{~nm}$. The bathochromic shift is consistent with its more conjugated planar structure due to the presence of the selenopheno[3,2-b]thiophene unit. The maximum fluorescence peak of BSeT2 was observed as blue emission at $468 \mathrm{~nm}$ with a shoulder at $491 \mathrm{~nm}$, which is largely blue shifted by $43 \mathrm{~nm}$ compared to BSeT1. In comparison to a cross conjugated selenopheno[2,3-b]thiophene possessing parent molecule, these results demonstrated a better intramolecular CT from conjugated selenopheno[3,2-b]thiophene unit to dimesitylborane in A$\pi-\mathrm{D}-\pi-\mathrm{A}$ system. ${ }^{25}$ The fluorescence quantum efficiency of BSeT1 was determined to be $\left(\Phi_{F}\right)$ of $11 \%$ slightly higher than that of BSeT2 $\left(\Phi_{F}=8 \%\right)$, which provides effective intramolecular charge transfer in $\pi$-conjugated system. In addition, compared to sulfur analogues ${ }^{25}$ these compounds have showed lower fluorescence quantum yields, because the fluorescence is quenched upon replacement sulfur with selenium atom. ${ }^{24}$

25

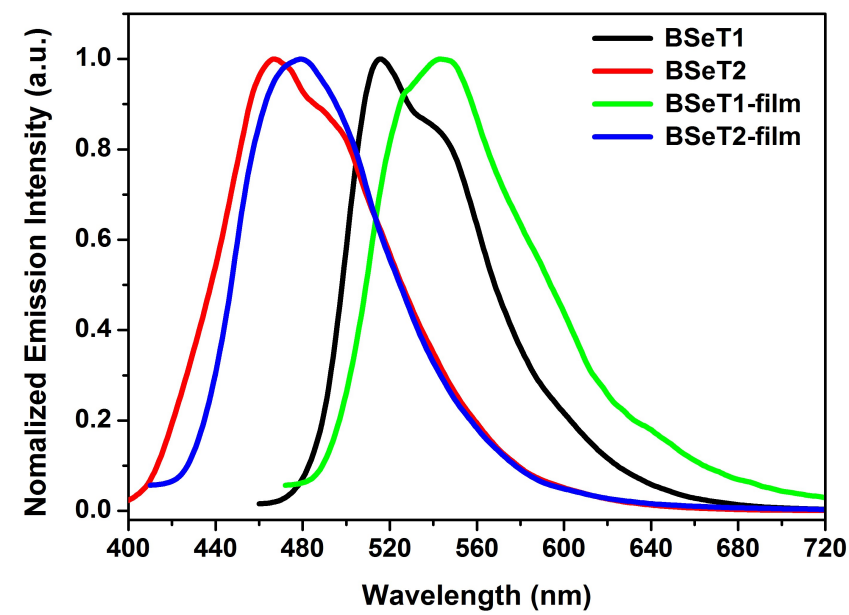

Figure 2. The fluorescence spectra of BSeT1 and BSeT2

However, the Stokes shift of BSeT1 was calculated to be $3200 \mathrm{~cm}^{-1}$, whereas the largest Stokes shift of $4400 \mathrm{~cm}^{-1}$ was obtained for BSeT2 in THF, which is highly expected for A- $-\pi-\mathrm{D}-\pi-\mathrm{A}$ systems containing dimesitylboryl units (Table 1). ${ }^{25-27}$ These results also mean that the excited-state 
dipole moment of BSeT1 is less than that of BSeT2, in which the dimesitylboron unit acts as a strong acceptor in the excited state.

In the solid state, maximum emission wavelengths of compounds are longer than those in solution. BSeT1 provided bright-green emission at $546 \mathrm{~nm}$ and BSeT2 displayed blue emission at 480 $\mathrm{nm}$. In comparison to the solution state, a clear red-shift ranging from 12 to $30 \mathrm{~nm}$ could be attributed to dimesitylboron acceptor and rigid fused SeT donor core units having better interactions in solid state. $^{25}$

\subsection{Electrochemical Properties}

Cyclic voltammetry measurements were conducted in $\mathrm{CH}_{2} \mathrm{Cl}_{2}$ at room temperature to obtain the electrochemical properties of the molecules. While BSeT1 (Figure 3) demonstrated one quasireversible wave at the positive potential of $0.76 \mathrm{~V}$ attributed to the oxidation of the SeT unit, BSeT2 displayed one irreversible wave $\left(\mathrm{E}_{\mathrm{ox}}=1.51 \mathrm{~V}\right)$ in oxidation potential (Figure 4). The reason for the higher oxidation potential of BSeT2 is weaker or no electronic communication as a result of the crossconjugated SeT unit.
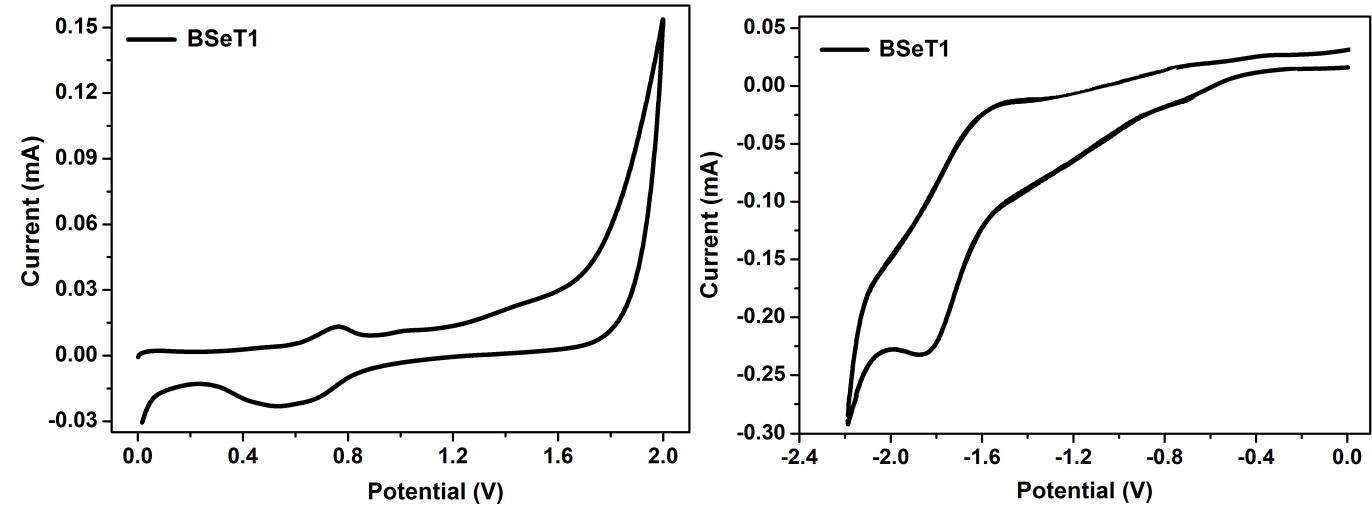

Figure 3. Cyclic voltammograms of $\mathbf{B S e T 1}$ in $\mathrm{CH}_{2} \mathrm{Cl}_{2}$ for oxidation and $\mathrm{ACN}$ for reduction potential, $0.1 \mathrm{M} \mathrm{Bu}_{4} \mathrm{NPF}_{6}, 100 \mathrm{mV} \mathrm{s}^{-1}$ scan rate, vs. $\mathrm{Fc} / \mathrm{Fc}^{+}$

The reduction potentials of BSeT1 and BSeT2 were investigated in ACN (Figures 3 and 4). For BSeT1, a single-step two-electron reduction process with one reversible redox wave at $\mathrm{E}_{\text {red }}=-$ $1.84 \mathrm{~V}$ vs. $\mathrm{Fc} / \mathrm{Fc}^{+}$(Figure 3) was observed indicating the formation of dianion, which demonstrated the two electrons accepted by the boron centres. ${ }^{25,29,30}$ However, BSeT2 showed a single-step twoelectron reduction process with one quasi-reversible peak at $\mathrm{E}_{\text {red }}=-1.96 \mathrm{~V}$ vs. $\mathrm{Fc} / \mathrm{Fc}^{+}$, which illustrated a better electron-accepting ability of BSeT1 with respect to BSeT2. Moreover, this result depicted a better $\pi$-conjugation arisen from the higher coplanarity and stronger electronic communication between the two boron centres through the rigid conjugated SeT spacer. In addition, the reduction potential of BSeT1 is more negative as compared to its thienothiophene analogue, ${ }^{25}$ pointing out the effect of SeT unit as an electron donor which slightly reduces the electron accepting ability of the boron centre. 

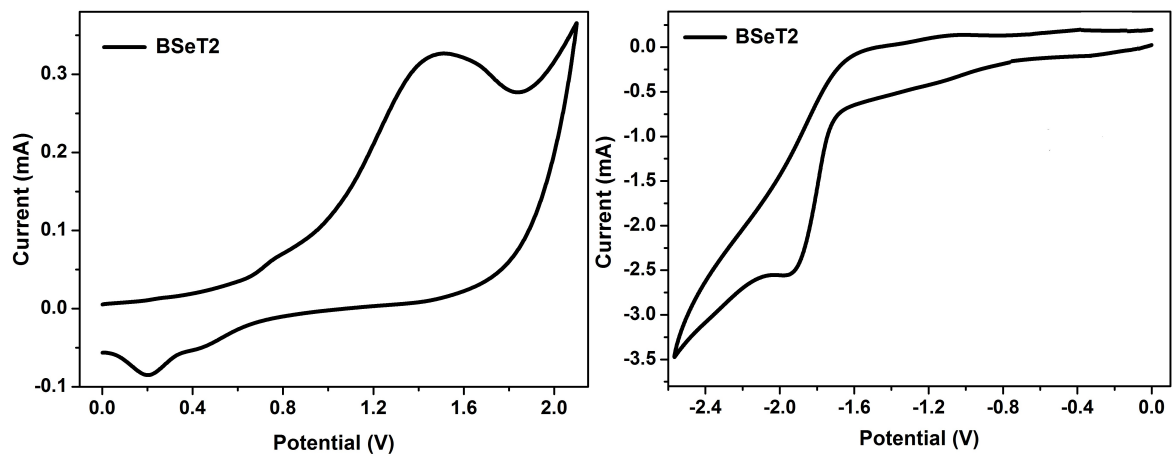

Figure 4. Cyclic voltammograms of $\mathbf{B S e T 2}$ in $\mathrm{CH}_{2} \mathrm{Cl}_{2}$ for oxidation and $\mathrm{ACN}$ for reduction potential, $0.1 \mathrm{M} \mathrm{Bu}_{4} \mathrm{NPF}_{6}, 100 \mathrm{mV} \mathrm{s}^{-1}$ scan rate, vs. $\mathrm{Fc} / \mathrm{Fc}^{+}$

The energies of frontier orbitals (HOMO and LUMO) of BSeT1 and BSeT2 were obtained from CV measurements (Table 1). The HOMO energies were calculated as -5.08 and $-5.29 \mathrm{eV}$, respectively, by using the onset potentials of the oxidations $\mathrm{E}_{\mathrm{ox}}{ }^{\text {onset }}=0.68 \mathrm{~V}$ for BSeT1 and $\mathrm{E}_{\mathrm{ox}}{ }^{\text {onset }}=0.89 \mathrm{~V}$ for BSeT2. The HOMO of BSeT1 is stabilized by $0.21 \mathrm{eV}$ indicating an efficient conjugation in BSeT1. The LUMO energy of BSeT1 $(-3.02 \mathrm{eV})$ is considerably lower than that of BSeT2 $(-2.84 \mathrm{eV})$. LUMOs are mainly located on the $\mathrm{p}_{\pi}$ orbital of the boron centre with significant contributions from the $\pi$-conjugated SeT system. The HOMO-LUMO gaps estimated electrochemically to be $2.06 \mathrm{eV}$ for BSeT1 and $2.45 \mathrm{eV}$ for BSeT2, which are well in alignment with the optical band gaps.

\subsection{Computational}

Density functional theory (DFT) calculations were conducted to shed light on the electronic and structure properties of compounds. Their gas phase geometry optimizations were performed without any symmetry constraints using Gaussian 09 package program. B3LYP $\mathrm{P}^{31-33}$ method was applied with Pople's medium basis set 6-31G(d) due to its good performance in optimization of structures and optoelectronic properties of the compounds. ${ }^{34}$ The minima were confirmed by analysing the harmonic vibrational frequencies using analytical second derivatives. TD-DFT calculations were realized using coulomb-attenuating density functional theory $(\mathrm{CAM}-\mathrm{TD}-\mathrm{B} 3 \mathrm{LYP})^{35}$ with 6$311+\mathrm{G}(\mathrm{d}, \mathrm{p})$ basis set to obtain the lowest singlet-singlet vertical excitations because CAM-B3LYP/6$311+\mathrm{G}(\mathrm{d}, \mathrm{p})$ rendered comparable results to the experimentally recorded excitation energies. ${ }^{36}$ Solvent (THF) was incorporated using polarizable continuum model (PCM) ${ }^{37}$ NStates of 50 for singlets were considered to obtain absorption bands. MOs were surfaced with an isosurface value of 0.03 au using GaussView 5.0 and absorption spectra with oscillator strengths were drawn with GaussSum 3.0. ${ }^{38}$

Computations performed on BSeT1 and BSeT2 illustrated that thiophenes attached to SeT units are deviating from the plane of SeTs by $5^{\circ}$ and $16^{\circ}$, respectively, depicting a good conjugation between $\mathrm{SeT}$ and thiophene groups (Figure 5). While thiophene $\pi$-spacers have dihedral angles of approximately $15^{\circ}$ with respect to the boron planes, the mesityl units attached to boron atom were estimated to be almost vertical to the plane of SeTs $\left(>59^{\circ}\right)$. HOMO is delocalized over T-SeT-T plane in BSeT1, whereas LUMO of the molecule is mainly localized on boron atoms spreading over the molecule between two boron atoms. However, while BSeT2 possessing a cross-conjugated system has the HOMO localized on SeT unit spreading over the thiophene substituents, the LUMO shows up as two fragments which are separated by SeT and mostly located on boron atoms with slight contributions of thiophene units in each side (Figure 5). The nodal plane on SeT in LUMO of BSeT2 causes the charge density separation and consequently hampers the delocalization of LUMO through the whole A-D-A system. MOs clearly indicate an efficient intramolecular charge transfer (ICT) between SeT donor and boron acceptor units. 


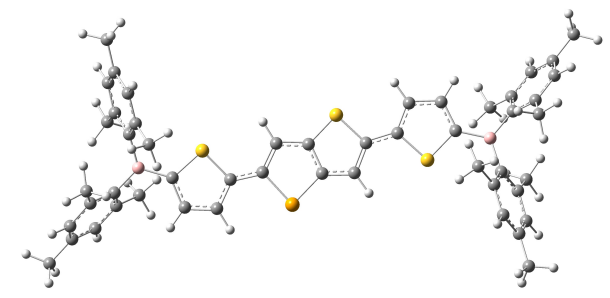

BSeT1

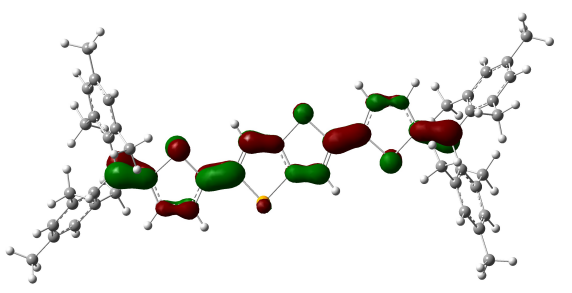

LUMO

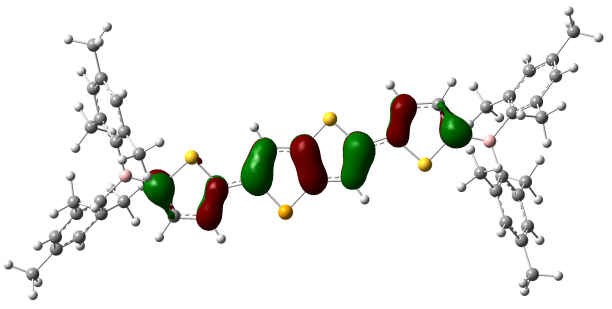

$\mathrm{HOMO}$

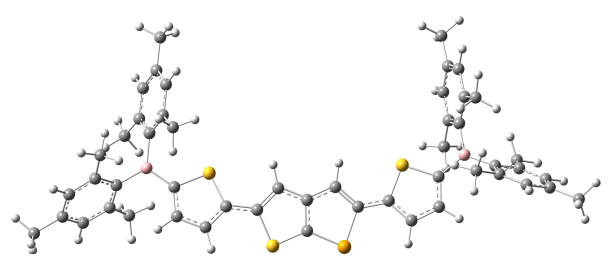

BSeT2

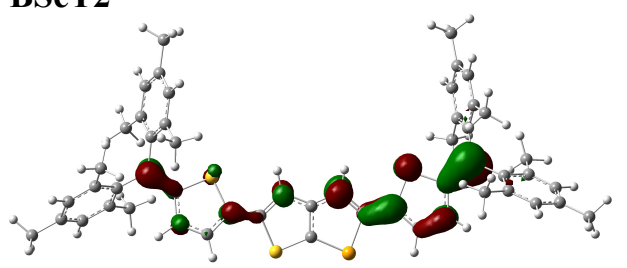

LUMO

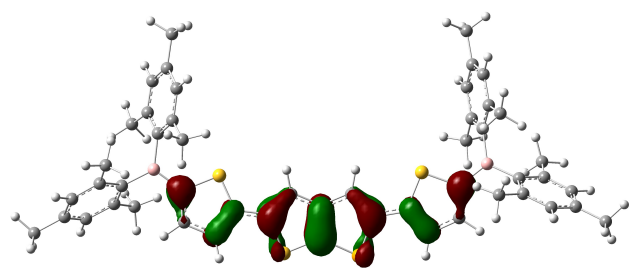

HOMO

Figure 5. HOMO and LUMO representations of BSeT1 and BSeT2 at B3LYP/6-31G(d) level (isosurface value: 0.4 a.u.)

Table 2. Excited state electronic transitions at (PCM:THF)-CAM-TD-B3LYP/6311+G(d,p)//B3LYP/6-31G(d) level.

\begin{tabular}{cccclc}
\hline Comp. & $\lambda_{\text {abs }}(\mathrm{nm})$ & $\mathrm{E}(\mathrm{eV})$ & $F^{\mathrm{a}}$ & \multicolumn{1}{c}{ Major contribution $^{\mathrm{b}}(\%)$} & Exp.(nm) \\
\hline \multirow{3}{*}{ BSeT1 } & 450 & 2.76 & 1.992 & $\mathrm{H} \rightarrow \mathrm{L}(91 \%)$ & 442 \\
& 312 & 3.97 & 0.128 & $\mathrm{H}-2 \rightarrow \mathrm{L}(48 \%), \mathrm{H}-2 \rightarrow \mathrm{L}+1(35 \%)$ & 340 \\
& 268 & 4.63 & 0.149 & $\mathrm{H}-12 \rightarrow \mathrm{L}(33 \%), \mathrm{H} \rightarrow \mathrm{L}+2(27 \%)$ & 274 \\
BSeT2 & 387 & 3.20 & 1.796 & $\mathrm{H} \rightarrow \mathrm{L}(76 \%)$ & 388 \\
& 364 & 3.41 & 0.255 & $\mathrm{H} \rightarrow \mathrm{L}+1(73 \%), \mathrm{H}-1 \rightarrow \mathrm{L}(16 \%)$ & 334 \\
\hline
\end{tabular}

${ }^{\mathrm{a}}$ Oscillator strength. ${ }^{\mathrm{b}} \mathrm{H}$ : HOMO, L: LUMO.

\section{Conclusion}

Two novel A- $\pi-\mathrm{D}-\pi-\mathrm{A}$ type compounds BSeT1 and BSeT2 were prepared using dimesitylboron as an electron acceptor with SeT analogues as electron donors and thiophene as a $\pi$ spacer. Their electrochemical and photophysical properties were investigated thoroughly. Conjugated and cross-conjugated SeTs influence their electronic behaviours. Conjugated BSeT1 showed considerable bathochromic shifts in both absorption and fluorescence spectra compared to the crossconjugated BSeT2. BSeT1 demonstrated a high electron affinity owing to the direct contribution of the vacant $\mathrm{p}_{\pi}$ orbital of the boron in the conjugate system lowering the LUMO energy level which endows BSeT1 with a promising electron-transport capability. Their estimated HOMO-LUMO gaps from cyclic voltammetry correlate well with those obtained from computational studies. These results 
might open a new avenue for D-A type molecular functionalization and potential applications in optical devices.

\section{Acknowledgements}

I would like to express my deep thanks to Prof. Turan Ozturk for his kind advice and valuable help. I am grateful to Asst. Prof. M. Emin Cinar for the suggestions about the computational studies. The Scientific and Technological Research Council of Turkey (TUBITAK) for the Postdoctoral Research Fellowship BIDEB 2218 program is gratefully acknowledged. I am indebted to National Centre for High Performance Computing (UYBHM) for the computer time provided and Unsped Global Logistic for financial support.

\section{Supporting Information}

NMR spectra, Computed absorption spectra and Cartesian coordinates. Supporting information accompanies this paper on http://www.acgpubs.org/OC

ORCID :

\section{References}

[1] Cinar, M. E.; Ozturk, T. Thienothiophenes, dithienothiophenes, and thienoacenes: syntheses, oligomers, polymers, and properties. Chem. Rev. 2015, 115, 3036-3140.

[2] Yamamoto, T.; Takimiya, K. Facile synthesis of highly $\pi$-extended heteroarenes, dinaphtho[2,3-b:2‘,3`f]chalcogenopheno[3,2-b]chalcogenophenes, and their application to field-effect transistors. J. Am. Chem. Soc. 2007, 129, 2224-2225.

[3] Dikcal, F.; Ozturk, T.; Cinar, M.E. Fused thiophenes: An overview of the computational investigations. Org. Commun. 2017, 10, 56-71.

[4] Arsenyan, P. A simple method for the preparation of selenopheno[3,2-b] and [2,3-b]thiophenes. Tetrahedron Lett. 2014, 55, 2527-2529.

[5] Patra, A.; Wijsboom, Y. H.; Zade, S. S.; Li, M.; Sheynin, Y.; Leitus, G.; Bendikov, M. Poly(3,4ethylenedioxyselenophene). J. Am. Chem. Soc. 2008, 130, 6734-6736.

[6] Lee, W-H.; Son, S. K.; Kim, K.; Lee, S. K.; Shin, W. S.; Moon, S-J.; Kang, I-N. Synthesis and characterization of new selenophene-based donor-acceptor low-bandgap polymers for organic photovoltaic cells. Macromolecules 2012, 45, 1303-1312.

[7] Heeney, M.; Zhang, W.; Crouch, D. J.; Chabinyc, M. L.; Gordeyev, S.; Hamilton, R.; Higgins, S. J.; McCulloch, I.; Skabara, P. J.; Sparrowe, D.; Tierney, S. Regioregular poly(3-hexyl)selenophene: a low band gap organic hole transporting polymer. Chem. Commun. 2007, 47, 5061-5063.

[8] Chung, D. S.; Kong, H.; Yun, W. M.; Cha, H.; Shim, H-K.; Kim, Y-H.; Park, C. E. Effects of selenophene substitution on the mobility and photovoltaic efficiency of polyquaterthiophene-based organic solar cells. Org. Electron. 2010, 11, 899-904.

[9] Das, S.; Zade, S. S. Poly(cyclopenta[c]selenophene): a new polyselenophene. Chem. Commun. 2010, 46, 1168-1170.

[10] Haid, S.; Mishra, A.; Uhrich, C.; Pfeiffer, M.; Bauerle, P. Dicyanovinylene-substituted selenophenethiophene co-oligomers for small-molecule organic solar cells. Chem. Mater. 2011, 23, 4435-4444.

[11] Turkoglu, G.; Cinar, M. E.; Buyruk, A.; Tekin, E.; Mucur, S. P.; Kaya, K.; Ozturk, T. Novel organoboron compounds derived from thieno[3,2-b]thiophene and triphenylamine units for OLED devices. J. Mater. Chem. C, 2016, 4, 6045-6053.

[12] Cheng, Y. J.; Yang, S. H.; Hsu, C. S. Synthesis of conjugated polymers for organic solar cell applications. Chem. Rev. 2009, 109, 5868-5923.

[13] Jäkle, F. Advances in the synthesis of organoborane polymers for optical, electronic, and sensory applications. Chem. Rev. 2010, 110, 3985-4022.

[14] Priegert, A. M.; Rawe, B. W.; Serin, S. C.; Gates, D. P. Polymers and the p-block elements. Chem. Soc. Rev. 2016, 45, 922-953.

[15] Ren, Y.; Jäkle, F. Merging thiophene with boron: new building blocks for conjugated materials. Dalton Trans. 2016, 45, 13996-14007.

[16] Turkoglu, G.; Cinar, M. E.; Ozturk, T. Triarylborane-based materials for OLED applications. Molecules, 2017, 22, 1522. 
[17] Brown, H. C.; Dodson, V. H. Studies in stereochemistry. XXII. The preparation and reactions of trimesitylborane. Evidence for the non-localized nature of the odd electron in triarylborane radical ions and related free radicals. J. Am. Chem. Soc. 1957, 79, 2302-2306.

[18] Yamaguchi, S.; Wakamiya, A. Boron as a key component for new $\pi$-electron materials. Pure Appl. Chem. 2006, 78, 1413-1424.

[19] Hudson, Z. M.; Wang, S. Impact of donor-acceptor geometry and metal chelation on photophysical properties and applications of triarylboranes. Acc. Chem. Res. 2009, 42, 1584-1596.

[20] Wade, C.R.; Broomsgrove, A. E. J.; Aldridge, S.; Gabbai, F. P. Fluoride ion complexation and sensing using organoboron compounds. Chem. Rev. 2010, 110, 3958-3984.

[21] Lorbach, A.; Hübner, A.; Wagner, M. Aryl(hydro)boranes: versatile building blocks for boron-doped $\pi$ electron materials. Dalton Trans. 2012, 41, 6048-6063.

[22] Stahl, R.; Lambert, C.; Kaiser, C.; Wortmann, R.; Jakober, R. Electrochemistry and photophysics of donor-substituted triarylboranes: symmetry breaking in ground and excited state. Chem. Eur. J. 2006, 12, 2358-2370.

[23] Zhao, C. H.; Sakuda, E.; Wakamiya, A.; Yamaguchi, S. Highly emissive diborylphenylene-containing bis(phenylethynyl)benzenes: structure-photophysical property correlations and fluoride ion sensing. Chem. Eur. J. 2009, 15, 10603-10612.

[24] Turkoglu, G.; Cinar, M. E.; Ozturk, T. Organoboron copolymers containing thienothiophene and selenophenothiophene analogues: optical, electrochemical and fluoride sensing properties. RSC $A d v$. 2017, 7, 23197-23207.

[25] Turkoglu, G.; Cinar, M. E.; Ozturk, T. Synthesis, photophysical and anion sensing properties of triarylborane-substituted cross-conjugated and conjugated thienothiophenes. Eur. J. Org. Chem. 2017, 31, 4552-4561.

[26] Entwistle, C. D.; Marder, T. B. Applications of three-coordinate organoboron compounds and polymers in optoelectronics. Chem. Mater. 2004, 16, 4574-4585.

[27] Doda, T.; Shirota, Y. 5,5'-Bis(dimesitylboryl)-2,2'-bithiophene and 5,5“'-bis(dimesitylboryl)$2,2^{6}: 5^{\circ}, 2^{\text {" }}$-terthiophene as a novel family of electron-transporting amorphous molecular materials. $J$. Am. Chem. Soc. 1998, 120, 9714-9715.

[28] Donat-Bouillud, A.; Lévesque, I.; Tao, Y.; D'Iorio, M.; Beaupré, S.; Blondin, P.; Ranger, M.; Bouchard, J.; Leclerc, M. Light-emitting diodes from fluorene-based $\pi$-conjugated polymers. Chem. Mater. 2000, 12, 1931-1936.

[29] Zhou, G.; Baumgarten, M.; Mullen, K. Mesitylboron-substituted ladder-type pentaphenylenes: chargetransfer, electronic communication, and sensing properties. J. Am. Chem. Soc. 2008, 130, 12477-12484.

[30] Zhao, S.-B.; Wucher, P.; Hudson, Z. M.; McCormick, T. M.; Liu, X.-Y.; Wang, S.; Feng, X.-D.; Lu, Z.$\mathrm{H}$. Impact of the linker on the electronic and luminescent properties of diboryl compounds: molecules with two $\mathrm{BMes}_{2}$ groups and the peculiar behavior of 1,6-(BMes$)_{2}$ pyrene. Organometallics 2008, 27, 6446-6456.

[31] Becke, A. D. A new mixing of Hartree-Fock and local density-functional theories. J. Chem. Phys. 1993, 98, 1372-1377.

[32] Becke, A. D. Densityfunctional thermochemistry. III. The role of exact exchange. J. Chem. Phys. 1993, 98, 5648-5652.

[33] Lee, C.; Yang, W.; Parr, R. G. Development of the Colle-Salvetti correlation-energy formula into a functional of the electron density. Phys. Rev. B 1988, 37, 785-789.

[34] Ravindran, E.; Ananthakrishnan, S. J.; Varathan, E.; Subramanian, V.; Somanathan, N. White light emitting single polymer from aggregation enhanced emission: a strategy through supramolecular assembly. J. Mater. Chem. C 2015, 3, 4359-4371

[35] Yanai, T.; Tew, D. P.; Handy, N. C. A new hybrid exchange-correlation functional using the Coulombattenuating method (CAM-B3LYP). Chem. Phys. Lett. 2004, 393, 51-57.

[36] Borges, I.; Uhl, E.; Modesto-Costa, L.; Aquino, A. J. A.; Lischka, H. Insight into the excited state electronic and structural properties of the organic photovoltaic donor polymer poly(thieno[3,4b]thiophene benzodithiophene) by means of ab initio and density functional theory. J. Phys. Chem. C 2016, 120, 21818-21826.

[37] Cossi, M.; Barone, V. Time-dependent density functional theory for molecules in liquid solutions. $J$. Chem. Phys. 2001, 115, 4708-4717.

[38] O'Boyle, N. M.; Tenderholt, A. L.; Langner, K. M. cclib: a library for package-independent computational chemistry algorithms. J. Comp. Chem. 2008, 29, 839-845.

[39] Jones II, G.; Jackson, W. R.; Choi C.; Bergmark, W. R. Solvent effects on emission yield and lifetime for coumarin laser dyes. Requirements for a rotatory decay mechanism. J. Phys. Chem. 1985, 89, 294-300. 
[40] Reynolds, G. A.; Drexhage, H. New coumarin dyes with rigidized structure for flashlamp-pumped dye lasers. Opt. Commun., 1975, 13, 222-225.

$$
\underset{\substack{\text { publications } \\ \text { (C) 2018 ACG Publications }}}{\text { A }}
$$

